\title{
EMOTIONS IN VIRTUAL REALITY
}

\author{
Darlene Barker and Haim Levkowitz \\ Department of Computer Science, \\ University of Massachusetts Lowell, Lowell, MA, USA
}

\begin{abstract}
One of the first senses we learn about at birth is touch, and the one sense that can deepen our experience of many situations is touch. In this paper we propose the use of emotions including touch within virtual reality (VR) to create a simulated closeness that currently can only be achieved with in-person interactions and communications. With the simulation of nonverbal cues, we can enhance a conversation or interaction in VR. Using haptic devices to deliver the simulation of touch between users via sensors and machine learning for emotion recognition based on data collected; all working towards simulated closeness in communication despite distance or being in VR. We present a direction for further research on how to simulate inperson communication within VR with the use of emotion recognition and touch to achieve a close-to-real interaction.
\end{abstract}

\section{KEYWORDS}

Virtual reality, emotion recognition, deep learning, in-personal communication simulation, nonverbal communication.

\section{INTRODUCTION}

To make an impact on social interaction within virtual reality (VR), we need to consider the effect of emotions in our in-person communications and their expression within VR [12]. The discussion "and theorizing about what makes up a virtual reality or virtual experience" has been going on since the 1960s and even further back when it was a dream or a work of fiction [6]. Now that virtual reality is a reality and VR headsets have become fixtures in a growing number of households, we need to utilize this technology to work towards a more impactful social interaction and establishing the connectedness we crave as humans [4].

One of the drawbacks of current forms of long-distance communication methods is that they lack the physical, nonverbal contact and expression that "form an important part of information exchange in our everyday interpersonal experiences" [2]. We rely on "realistic sensory simulations" for visual, tactile, and audio cues [9]. What we are proposing is simulating this in VR with the use of emotions. There are four pieces to the puzzle of making a long-distance interaction feel intimate within VR-emotions, voice, facial expressions, and touch. We express our emotions in day-to-day communications using the other three pieces. To do the same in VR, we need a novel way of implementing touch. Now, the goal becomes making the experience as close to the real world as possible, while also keeping it interesting and achievable.

When a person speaks, a lot can be interpreted from the acoustic properties of what they said; these can be features that are recorded and used to help interpret their emotional state. This can include the pitch at which they spoke and how loud they were. Facial expressions are changes in facial muscles that can show emotions, including the basic ones, such as, happy, sad, surprise, David C. Wyld et al. (Eds): CSTY, SIGI, MaVaS - 2021 
fear, anger. This happens when "a series of biological events follow that produce changes in a person" [1]. You are happy to see the other person on the line and you smile with your whole face. That is a favorable reaction but then let us say, you are receiving disappointing news: your facial reaction will be quite different.

The sense of touch is one of those senses that a newborn can experience for the first time right out of the womb with their mother. It is "the first of our senses to develop, and it provides us with our most fundamental means of contact with the external world" making this an important part of emotion and the sense of presence in the real world [2]. So, in trying to recreate this in VR, touch becomes a critical part of the formula of an immersive experience. Not only does touch affect our emotions, but it also affects how we act, make connections to, and interact with others.

During in-person interactions, we use verbal and nonverbal body language cues to communicate. Long-distance communication like text messages, phone calls, video calls, and handwritten letters will only offer part of the whole experience. Within a virtual environment (VE), we can make long-distance communications closer to in-person experiences and add a level of intimacy using emotion recognition and touch utilization. We propose "smart clothes" along with head mounted displays to create such a virtual environment [3]. At this time, we can only provide feedback on the controllers for hand holding and headsets that are currently available. However further research is needed for the entire body. Being able to see the face of the person and their body movements, along with the added feature of touch, creates a greater degree of intimacy in long-distance communication.

"Virtual reality (VR) allows for a close approximation of the real world," which is what we are using to create an intimate long-distance communication within a "controllable approximation of the real, physical world" [8]. We propose to use VR for long-distance communications where the avatars of the parties reflect their emotions, and each party can touch each other. When they touch, they will receive a signal and have an emotional response that will be visible to the party initiating the touch. The emotions create a sense of presence that helps with the immersive element of this type of communication.

\section{OUR APPROACH}

We are proposing the use of passive sensors to capture physiological changes during interaction in a virtual environment as well as sensors that capture deliberate actions on either party. So, one party reaches out to touch the arm of the other party; the sensors would collect data and that data would assist in the simulation of the feeling of being touched by the other party. This would happen in real time for both parties. When you touch a person's arm, you feel the arm and at same time, you also feel a response. The other party feels this touch and may or may not respond actively. So, this is where the passive sensors can be of assistance. An example could be feeling a cold object on your arm. One could respond with that was not cold, but the arm shivers a tiny bit; and delivers a response that would have been seen if you were next to the person. We are aiming towards a more real simulation of touch and emotional interaction in VR.

The idea is to create a complete environment "or world" for the users to experience a close interaction with each other even if they are separated by distance [6]. We are proposing "a unique interaction space where users can perceive a different reality" than their own, one in which distance does not affect the interaction as it does in the real world [6]. The idea is having two users interact in real time in VR in a computer-generated VE. Instead of using objects in the world to stimulate the user's emotions and affect their sense of presence, we are proposing the capture of their emotions from an evaluation of their speech, facial expression, physical changes in their body, body language, and how they are interacting with the world [7]. 
We propose doing this using smart garments and other VR wearable devices that can read input and transfer signals to and from the wearer of the garment or device [3], [11], [21]. This is one of the goals of this research. While VR can be seen as having a need "to create a sense of 'connection' to evoke feelings of presence and enhance user engagement", we are aiming for a simulation of the physical experience [23]. Current VR experiences "touch illusion relying on visuals and sound is thought to increase immersion", we are aiming at simulating touch which will bring us closer to the mark in recreating emotions in VR [21], [23].

One may ask, why touch? Well, it is the first sense that we develop as an infant. Often, our first experience in the world is the touch of the mother. Touching an object can make the experience more real than simply looking at it; it gives the connectedness that we want in VR which will immerse the user in the virtual world. "The sense of touch is a collection of several sensations, encompassing pressure, pain and temperature, and touch receptors are stimulated by a combination of mechanical, chemical and thermal energy" [11]. By capturing those sensations along with the physiological changes in the body, we are aiming to add touch to the experience in VR "to deliver something that can approximate to realistic interpersonal touch" [2].

Much of the data collection will need to be evaluated in context since data will be collected from multiple sensors because of many changes that occur in our bodies for a single emotion to be expressed [17]. To then recreate an emotion such as happiness; the eyes are wide open, there may be some excitement, the palms a little sweaty, the face may be smiling, the back may be straight, and the upper body may be open and confident, and the voice may be high pitched. All of these depend on the baseline of the users.

Data collection could include the use of cameras within the headset and outside to capture facial expressions potentially including eye-tracking, movements of the body, and any body language cues [16], [18]. Other data collection could include an EEG sensor to capture brain waves, and other sensors collecting the temperature of the skin, galvanic skin response (GSR), speech, eyetracking, heart rate [13], [18], [19], [20]. Speech can be captured and transmitted but there can be patterns in the way things are being said that could give a clearer picture of the emotional state of the spoken part. We will use techniques from machine learning to process this data that we collect on both parties and recreate each party visually (avatar) as well as the feelings associated with touch [18], [22]. The idea will be to produce an avatar that matches the emotional state of the person the data was collected from without any input from the person being captured.

One of the techniques that is being considered is a LSTM based deep learning network to assist in detecting the emotions, as well as the effect of what one is hearing in the virtual environment, such as music, or other sounds including the speech of the other party [14], [15].

Table 1. Communication options and senses used

\begin{tabular}{|c|c|c|c|}
\hline \multirow{2}{*}{$\begin{array}{c}\text { Long-distance } \\
\text { communication Options }\end{array}$} & \multicolumn{3}{|c|}{ Senses } \\
\hline & Hearing & Sight & Touch \\
\hline Phone calls & Yes & & \\
\hline Emails & & & \\
\hline Video chat apps & Yes & Yes & \\
\hline Handwritten letters & & & \\
\hline VR Chat & Yes & Yes & \\
\hline Virtual Touch & Yes & Yes & Yes \\
\hline
\end{tabular}


In Table 1 we see communications options and senses that we utilize. Of the senses being covered in communication, hearing and sight are the ones covered most, and we are proposing to improve communications by adding touch to the mix through indirect observation via the use of passive sensors. We will create a VE setting and use emotions to help immerse the user into the world and be able to physically feel feedback beyond the feedback of a vibrating controller. We propose the use of emotion tracking which is used to interpret what both parties are feeling and deliver that to each other along with simulating touch to enhance the experience and what is being communicated.

\section{RELATED WORK}

The closest option to what we are trying to accomplish is the use of virtual interpersonal touch with expression and recognition of emotions using force-feedback haptic. Emotions can be calibrated from facial expression data detected from facial anchor points using machine learning, digitized audio streams, and data collected from virtual interpersonal touch [1]. Emotion data can be in the form of a 2D scale of the hedonic valences and intensity. Facial expression shows emotion in the form of the facial muscles and change of the heart rate or blood pressure. Voice can be gotten from digital audio streams.

Touch tends to increase trust in face-to-face communication, and it usually adds sincerity, establishes trust, weight, urgency, marks significance, or with greetings (shake hands). It is used in one-to-one interactions, seen as inefficient and inconveniently requiring proximity [1]. It was also found that "overly intimate or socially inappropriate for many interactions", "little is known about the psychological effects of haptic communication", "can create transformed haptic communication scenarios that are not possible in the physical world", and "communication of emotion in virtual reality does not necessarily have to copy the real world; instead, it can be abstracted" [1].

This study was found to not be as accurate as interpersonal handshakes and inefficient because the experiment required the subject to be connected to multiple devices. Touch was found to be too intimate if not socially inappropriate in some cases, but at the same time they were able "to convey emotions via a short interaction such as a handshake" [1].

For our approach, more than a handshake is necessary. We are trying to convey intimacy across distance. The idea is to be able to touch the hand of the other person as you would while having dinner or touch the arm or shoulder or some other show of affection.

Another paper by Gallace and Spence, talks about interpersonal touch and how it can be used to communicate emotion, improve our wellbeing, to share our feelings with others, and to enhance the meaning of other forms of verbal and non-verbal communication [2]. They also mention the "recent growth of studies investigating the addition of tactile sensations to long-distance communication technologies" in the form of "mediated or "virtual' touch" [2]. For our approach touch is an integral part that is missing from current virtual and long-distance communication options.

Another paper that included experiments (shown below) with the use of facial expressions and the use of touch, and in this case, a tactile glove is used for participants to be able to shake hands. This study found that "not only does touch affect emotional processes, but the processing of touch may also be" altered by other factors and may not be a positive emotion [4]. This does not cover what we are proposing in the executing of touch but does cover capturing other emotions. 


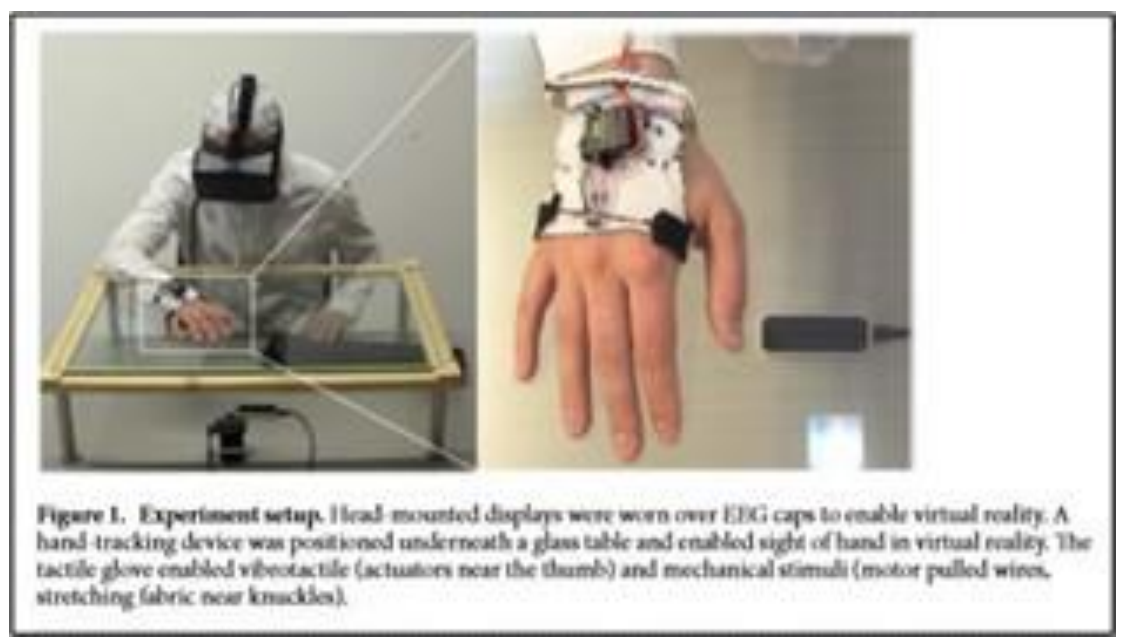

Figure 1. taken from Ravaja, N. et al [4]

We propose to do so using senses versus visual manipulation. As humans we are aware of our surroundings based on what we see, touch, smell, and feel. With the ability to touch and feel feedback from the virtual environment, we would have a more realistic sense of presence.

Another paper looked at altering the mood of the participants using touch and smell. The touch component was objects in the world, such as grass. There was a change to the person in the real world that affected their mood in the virtual world. They found that "the more senses stimulated, the higher the sense of presence" for the participants in the study [7]. For our approach, we are looking at creating the stimulation within the virtual environment.

In [10], Hinkle proposed a machine learning approach to extracting physical measurements "to determine emotional state of human test subjects through the non-invasive collection and analysis of physiological data during subject interaction virtual reality or other immersive audio/visual environments". Their approach proposes "a circumplex model of affect, more widely known as the arousal-valence model" using "image processing and machine learning techniques" where they were about to "classify emotion based on facial images and voice recordings" [10]. Our approach is to also collect the data with non-invasive means as well as machine learning to extract the features.

In [11], the authors discuss a current technology used for enhanced touch, a "skin-integrated technology that applies pressure, vibration or motion to the user, enabling communication between the user and a machine for virtual and augmented reality," which "have some drawbacks. For instance, each actuator is driven by a set current of about five milliamps, which is relatively high compared with that found in other consumer digital electronics" [11]. Each actuator is "equipped with embedded sensors that detect and encode the strength, duration and location of the touch, together with skin warmth and heart rate of the sender" [11]. This is the closest to what we are proposing but further research is needed.

\section{Potential Impact on Society}

This is an opportunity to make social interactions in VR more real since the addition of touch and the integration of emotions in the whole experience will add a level of accountability. This could make for more honest interaction in VR. This creates a new and viable means to maintain 
personal relationships when used for communication between parties who are separated by distance for extended periods of time.

Other areas that this could be applied to include gaming, prosthetics, and stress management. In gaming interactions in VR, it could add to the immersive nature of the technology. This could potentially be of a significant help in "prosthetic feedback" [11]. In the case where VR is being used to teach emotion recognition, a way to integrate touch could be impactful to the immersive requirement of the experience. This can be a tool for stress reduction since a "tender caress from a caregiver or spouse reduces stress and promotes emotional well-being" [5]. The stress of separation from loved ones can be reduced with the use of touch and emotion recognition in VR.

\section{FUTURE WORK}

The perceptual immersive nature of VR with the use of voice, face, and touch to execute a longdistance communication experience could give the illusion of being a step closer towards a nonverbal intimacy and a certain degree of emotional warmth. The reasoning for using touch in VR is establishing trust in interpersonal interactions gives the illusion of the closeness that is a necessary part of maintaining connections when separated by great distances.

VR has been around for some time and now it is becoming a fixture in a growing number of households; the next logical step would be to have it evolve into a tool that can help the human experience. VR can help communication across continents and great distances, provided the necessary power and Wi-Fi is available, and assist with maintaining the closeness that is lacking in current video, telephone, email, or text communication - which is all that is afforded in the current market.

Its not just VR but simulating touch via electronic technology can have many applications - say a visually impaired person can feel the skin of a loved one across the globe, as in:

- In our current normal, holding the hand of a loved one in a situation where physical contact cannot always happen, as in a medical facility, during this time of quarantine.

- Being able to be there for a loved one despite distance or circumstances, as in soldiers being deployed or a parent, or loved one away for work being able to experience important moments in our lives, such as, birth of a child, or some other special occasion. Being able to hold your partner, parent, or child's hand in those important moments despite being separated because of the pandemic or distance.

Granted, this approach requires further research on technology to make touch detection and transfer between two parties available to the consumer market, and integration with current VR head mounted devices. With all the above, this is still a novel idea to further the human experience.

\section{CONClusion}

Our goal is to bring people together despite distance in such a way that replicates and even rivals in-person communication. It could be used in video games, to enrich personal relationships, virtual healthcare services, virtual education, retail, therapeutic services, and potentially in other situations where physical interaction is not possible. 


\section{REFERENCES}

[1] Bailenson, J., Yee, N., Brave, S., Merget, D., Koslow, D., "Virtual Interpersonal Touch: Expressing and Recognizing Emotions Through Haptic Devices," Human-Computer Interaction, 2007, Volume 22, pp. 325-353.

[2] Gallace, A., Spence, C., "The science of interpersonal touch: An overview", Neuroscience and Biobehavioral Reviews, 34, 2010, pp. 246-259.

[3] Makki, I., Alhalabi, W., Adham, R., "Using Emotion Analysis to Define Human Factors of Virtual Reality Wearables", ScienceDirect, Procedia Computer Science 165, 2019, pp. 154-164.

[4] Ravaja, N., Harjunen, V., Ahmed, I., Jacucci, G., Spape, M., "Feeling Touched: Emotional Modulation of Somatosensory Potentials to Interpersonal Touch", Scientific Reports 7:40504, 2017, pp. 1-11.

[5] Harjunen, V. J., Spape, M., Ahmed, I., Jacucci, G., Ravaja, N., "Individual differences in affective touch: Behavioral inhibition and gender define how an interpersonal touch is perceived", Personality and Individual Differences, 107, 2017, pp. 88-95.

[6] Cisners, Angela, Maravilla, Morelia, Murray, Brian, Scretching, Desiree, Stoddard, Alvie, Redmiles, Elissa M., "Defining Virtual Reality: Insights from Research and Practice", iConference 2019 Proceedings, 2019.

[7] Serrano, B., Banos, R., Botella, C., "Virtual reality and stimulation of touch and smell for inducing relaxation: A randomized controlled trial”, Computers in Human Behavior 55, 2016, pp. 1-8.

[8] van der Ham, I., Klaassen, F., Van Schie, K., Cuperus, A., "Elapsed time estimates in virtual reality and the physical world: The role of arousal and emotional valence", Computers in Human Behavior 94, 2019, pp. 77-81.

[9] Lecuyer, Anatole, "Playing with Senses in VR: Alternate Perceptions Combining Vision and Touch", Spatial Interfaces 0272-1716, 2017, pp. 20-26.

[10] Hinkle, Lee B., Roudposhti, Kamrad K., Metsis, "Vangelis, Physiological Measurement for Emotion Recognition in Virtual Reality", International Conference on Data Intelligence and Security, 2019, pp. 136-143.

[11] Tao, Xiao-ming, "Virtual and augmented reality enhanced by touch", Nature, Vol. 575, 2019, pp. 453-454.

[12] Barker, Darlene, Levkowitz, Haim, "Tutorial 5: Emotions in Virtual Reality", IEEE VR 2021 Conference, https://ieeevr.org/2021/program/tutorials/\#T5

[13] Yang, Xiuleng, Emma McCoy, Esther Anaya-Boig, Ione Avila-Palencia, Christian Brand, Glòria Carrasco-Turigas, Evi Dons, et al, "The Effects of Traveling in Different Transport Modes on Galvanic Skin Response (GSR) as a Measure of Stress: An Observational Study", Environment International 156 (November), 2021, 106764.

[14] Sakalle, Aditi, Pradeep Tomar, Harshit Bhardwaj, Divya Acharya, and Arpit Bhardwaj, "A LSTM Based Deep Learning Network for Recognizing Emotions Using Wireless Brainwave Driven System", Expert Systems with Applications 173 (July), 2020, 114516.

[15] S. Sheykhivand, Z. Mousavi, T. Y. Rezaii and A. Farzamnia, "Recognizing Emotions Evoked by Music Using CNN-LSTM Networks on EEG Signals", IEEE Access, Vol. 8, 2020, pp. 139332139345.

[16] Geraets, C.N.W., S. Klein Tuente, B.P. Lestestuiver, M. van Beilen, S.A. Nijman, J.B.C. Marsman, and W. Veling, "Virtual Reality Facial Emotion Recognition in Social Environments: An EyeTracking Study", Internet Interventions 25 (September), 2021, 100432.

[17] B. Meuleman and D. Rudrauf, "Induction and Profiling of Strong Multi-Componential Emotions in Virtual Reality", IEEE Transactions on Affective Computing, Vol. 12, no. 1, Jan.-March 2021, pp. 189-202.

[18] Magdin, Martin, Zoltán Balogh, Jaroslav Reichel, Jan Francisti, Stefan Koprda, and Molnár György, "Automatic Detection and Classification of Emotional States in Virtual Reality and Standard Environments (LCD): Comparing Valence and Arousal of Induced Emotions", Virtual Reality 25, No. 4 (December 1, 2021): 1029.

[19] Marín-Morales, Javier, Juan Luis Higuera-Trujillo, Jaime Guixeres, Carmen Llinares, Mariano Alcañiz, and Gaetano Valenza, "Heart Rate Variability Analysis for the Assessment of Immersive Emotional Arousal Using Virtual Reality: Comparing Real and Virtual Scenarios." PLoS ONE 16, No. 7 (July 2021): 1-16. 
[20] Gilpin, Gina, James Gain, and Gosia Lipinska, "The Physiological Signature of Sadness: A Comparison between Text, Film and Virtual Reality", Brain and Cognition 152 (August 1, 2021).

[21] Yu, X., Xie, Z., Yu, Y. et al, "Skin-integrated wireless haptic interfaces for virtual and augmented reality", Nature 575, 473-479 (2019).

[22] Jianhua Zhang, Zhong Yin, Peng Chen, Stefano Nichele, "Emotion recognition using multi-modal data and machine learning techniques: A tutorial and review", Information Fusion, Volume 59, 2020, pp 103-126.

[23] Price, Sara \& Jewitt, Carey \& Yiannoutsou, Nikoleta, "Conceptualising touch in VR". Virtual Reality, 25, 2021, 10.1007/s10055-020-00494-y.

\section{AUTHORS}

Darlene Barker is a PhD Candidate in the Computer Science Department at the University of Massachusetts Lowell, in Lowell, MA, USA. She completed her master's degree in Computer Science from University of Massachusetts. She also completed her Bachelor of Science majoring in Computer Science from Rivier University in Nashua, NH. Her professional career experience spanning several years and disciplines from systems to software working for companies-Compaq Computer Corporation, Teradyne, Inc, and Oracle Corporation. She is the founder of

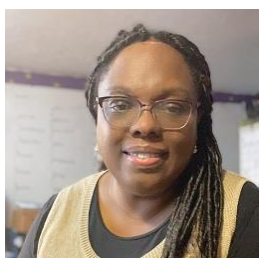
an independent video game studio, DB Attic Studios, LLC, in Bedford, NH where she makes video games with her children from middle school through high school graduates.

Haim Levkowitz is the Chair of the Computer Science Department at the University of Massachusetts Lowell, in Lowell, MA, USA, where he has been a faculty member since 1989. He was a twice-recipient of a US Fulbright Scholar Award to Brazil (August - December 2012 and August 2004 - January 2005). He was a Visiting Professor at ICMC - Instituto de Ciencias Matematicas e de Computacao (The Institute of Mathematics and Computer Sciences) - at the University of Sao Paul, Sao Carlos - SP, Brazil (August 2004 - August 2005; August 2012 to August 2013). He

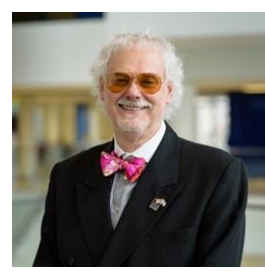
co-founded and was Co-Director of the Institute for Visualization and Perception Research (through 2012). $\mathrm{He}$ is a world-renowned authority on visualization, perception, color, and their application in data mining and information retrieval. He is the author of "Color Theory and Modeling for Computer Graphics, Visualization, and Multimedia Applications" (Springer 1997) and co-editor of "Perceptual Issues in Visualization" (Springer 1995), as well as many papers in these subjects. He is also co-author/co-editor of "Writing Scientific Papers in English Successfully: Your Complete Roadmap," (E. Schuster, H. Levkowitz, and O.N. Oliveira Jr., eds., Paperback: ISBN: 978-8588533974; Kindle: ISBN: 8588533979, available now on Amazon.com: http://www.amazon.com/Writing-Scientific-Papers-EnglishSuccessfully/dp/8588533979). He has more than 48 years-experience teaching and lecturing, and has taught many tutorials and short courses, in addition to regular academic courses. In addition to his academic career, Professor Levkowitz has had an active entrepreneurial career as Founder or Co-Founder, Chief Technology Officer, Scientific and Strategic Advisor, Director, and venture investor at several hightech start-ups.

(C) 2021 By AIRCC Publishing Corporation. This article is published under the Creative Commons Attribution (CC BY) license. 